\title{
"Comparison of the toxicity profile of PD-1 versus PD-L1 inhibitors in non-small cell lung cancer": is there a substantial difference or not?
}

\author{
Alessia Spagnuolo, Cesare Gridelli \\ Division of Medical Oncology, 'S. G. Moscati’ Hospital, Avellino, Italy \\ Correspondence to: Cesare Gridelli. Division of Medical Oncology, 'S. G. Moscati’ Hospital, Avellino, Italy. Email: cgridelli@libero.it. \\ Provenance: This is an invited Editorial commissioned by the Executive Editor-in-Chief Jianxing He (Department of Cardiothoracic Surgery, The \\ First Affiliated Hospital of Guangzhou Medical University, Guangzhou, China). \\ Comment on: Pillai RN, Behera M, Owonikoko TK, et al. Comparison of the Toxicity Profile of PD-1 Versus PD-L1 Inhibitors in Non-Small Cell \\ Lung Cancer: A Systematic Analysis of the Literature. Cancer 2018;124:271-7.
}

Submitted Sep 10, 2018. Accepted for publication Sep 17, 2018.

doi: $10.21037 /$ jtd.2018.09.83

View this article at: http://dx.doi.org/10.21037/jtd.2018.09.83

Metastatic non-small cell lung cancer (NSCLC) is still an incurable disease, despite the significant improvement in prognostic outcomes [progression-free survival (PFS) and overall survival (OS)] which has been seen in recent years following the advent of immunomodulatory drugs aimed at inhibiting immune checkpoints. The objectives of such active immunotherapy, which acts directly on the immune system and thus indirectly on the tumour, include enhancement of tumour-specific immunity, increased recruitment of effector cells at the tumour site and blockade of tumoral immune evasion strategies. One mechanism which helps tumour cells to avoid immune attack is the overexpression of programmed death-ligand 1 (PD-L1), the principal ligand of programmed death-1 (PD-1), an immune checkpoint receptor present on activated $\mathrm{T}$ lymphocytes and a co-inhibitory molecule, which under normal conditions limits the excessive activation of $T$ cells and thus protects normal cells during chronic inflammation and prevents autoimmune reactions $(1,2)$. Nivolumab and pembrolizumab (PD-1 inhibitors), and atezolizumab, durvalumab and avelumab (PD-L1 inhibitors), monoclonal antibodies shown to be effective in NSCLC treatment, are able to block the interaction between PD-1 and PDL1 and thus allow T lymphocytes to neutralise tumour cells. Naturally these drugs are associated with a unique set of toxic effects, which are different from those seen with conventional cytotoxic chemotherapy and are related to the reduction in auto tolerance resulting from loss of $\mathrm{T}$ cell inhibition $(3,4)$. They may involve any system of the body, and while they are generally manageable they can be fatal in some cases $(5,6)$. As the number of immune checkpoint inhibitors (ICIs) is constantly growing, a comprehensive understanding of the toxicity of immunotherapy agents is of great interest to oncologists when it comes to making an informed choice of treatment. The article by Pillai et al. entitled 'Comparison of the Toxicity Profile of PD-1 Versus PD-L1 Inbibitors in Non-Small Cell Lung Cancer: A Systematic Analysis of the Literature', describes a metaanalysis carried out with the Comprehensive MetaAnalysis software (version2.2) on studies of PD-1 and PD-L1 inhibitors (nivolumab and pembrolizumab, and atezolizumab, durvalumab and avelumab respectively) used as single agents in advanced NSCLC patients, published between 2000 and 2016. Relevant abstracts from the American Society of Clinical Oncology (2011-2016), the International Association for the Study of Lung Cancer World Conference on Lung Cancer (2011-2016) and the European Society for Medical Oncology (2011-2016) were also included (7). The following were excluded from the analysis: phase I studies, studies with fewer than 10 enrolled patients, studies in which ICIs were not used as monotherapy, studies which did not report toxicity data and retrospective studies. The primary outcome of the analysis was represented by toxicities, evaluating possible differences in safety and tolerability between PD-1 and PD-L1 inhibitors. Of 549 studies identified, 23 were 
included in the analysis, 12 using PD-1 inhibitors (8 with nivolumab and 4 with pembrolizumab) and 11 using PDL1 inhibitors (6 with atezolizumab, 3 with durvalumab and 2 with avelumab). Data from a total of 5,744 patients $(3,284$ with PD-1 inhibitors and 2,460 with PD-L1 inhibitors) with comparable basal characteristics in terms of median age, smoking history and ECOG Performance Status were employed in the evaluation of ICIs toxicity. The percentage of male patients in the two groups was different (59\% and $56 \%$ in the PD-1 and PD-L1 inhibitor cohorts respectively; $\mathrm{P}=0.4$ ), as was the number of patients with squamous cell lung cancer ( $25 \%$ in the PD- 1 inhibitor cohort and $32 \%$ in the PD-L1 inhibitor cohort; $\mathrm{P}=0.6$ ). In addition to the primary outcome, the analysis also included the overall response rate, for which no apparent difference emerged between the groups treated with PD-1 and PD-L1 inhibitors (19\% and $18.6 \%$ respectively; $\mathrm{P}=0.17)$. Regarding the main objective of the authors, the toxicity profiles of the anti-PD-1 and anti-PD-L1 monoclonal antibodies appear to be comparable, with overall adverse event (AE) incidences of $64 \%$ and $66 \%$ in patients treated with $\mathrm{PD}-1$ and PD-L1 inhibitors, respectively $(\mathrm{P}=0.8)$. However, $13 \%$ of patients undergoing treatment with anti-PD-1 agents and $21 \%$ of those given anti-PD-L1 agents developed AEs of grades 3 to $5(\mathrm{P}=0.15)$. The rate of immune-related AEs (irAEs) resulting from treatment with PD-1 inhibitors was $16 \%$, and that seen with PD-L1 inhibitors was $11 \%$ $(\mathrm{P}=0.07)$, although the incidences of grade $3-5$ irAEs in the two groups were $3 \%$ and $5 \%$ respectively $(\mathrm{P}=0.4)$. The most common general AE was fatigue (19\% in the group treated with PD-1 inhibitors, compared to $21 \%$ in the PDL1 inhibitor group; $\mathrm{P}=0.4)$, while hypothyroidism was the most frequently observed irAE $(6.7 \%$ and $4.2 \%$ in the anti-PD-1 and anti-PD-L1 groups respectively; $\mathrm{P}=0.07$ ). It is noteworthy that patients receiving PD-1 inhibitors experienced a higher incidence of pneumonitis than those who were given PD-L1 inhibitors ( $4 \%$ vs. $2 \%$; $\mathrm{P}=0.01$ ). The authors conclude that the toxicities of the two classes of ICIs are not dissimilar, with a therapeutic index superior to that seen in chemotherapy, and that the differences which do exist (apart from the pneumonitis data) are relatively minor.

This meta-analysis is an important work, based on a large set of data from prospective trials, but as these range from phase I to phase IV ( 8 phase I, 2 phase I/II, 6 phase II, 6 phase III and 1 phase IIIb/IV, with PD-L1 inhibitors used mainly in the early-stage trials), they are very different from one another, being designed with different objectives which rightly do not always include drug safety, at least not as a primary endpoint. Both randomised and single arm non-randomised trials were analysed (17 single arm and 6 randomised controlled trials), resulting in a predominance of single arm open-label trials at high risk of bias, mainly involving PD-L1 inhibitors, which showed dose escalation of single drugs in some cases, used the same agent at different doses, and employed different followup periods in different studies. Naturally, the inclusion criteria also varied between one study and another, and thus the patients enrolled differed, for example in terms of previous therapies, which obviously led to a difference in ability to tolerate treatment between treatment-naïve and previously treated patients. The analysis included 3 studies of first line treatment with PD-1 inhibitors and 2 such studies with PD-L1 inhibitors. Hence the majority of the patients studied were treated with PD-1 inhibitors used as a further line of treatment. Durvalumab, that appeared to be the agent most associated with toxicity, was evaluated in a limited number of subjects. Important points to consider are that discontinuation rates were not consistently described in the studies include in the analysis, and the precise number of patients experiencing grade 3-5 toxicity (influenced by the discontinuation rate) or AEs resulting in death was not always available. The authors underline that further potential causes of bias could be that in some cases the toxicity data were limited and taken from conference presentations, and that PD-1 inhibitors have been studied for longer time and are the subject of more extensive publications with more complete data. Toxicities are described in the analysis using versions 3 or 4 of the National Cancer Institute Common Terminology Criteria for Adverse Events (CTCAE), but the definition of a toxic effect as correlated to treatment is certainly not always an objective fact, and not all of the trials included in the analysis indicated AEs as correlated to treatment. Furthermore, the diagnosis of an AE is not always straightforward, particularly in the case of irAEs such as an endocrinopathy with non-specific symptoms (weight loss, fatigue, headache etc.), or of pneumonitis, which remains a diagnosis of exclusion between neoplastic infiltration and infection because defining pathognomonic criteria are lacking, even radiologically $(8,9)$. It is therefore important that uniform criteria of identification are employed in future studies in order to avoid under- or overestimation of signs and symptoms. There is recent evidence that the onset of irAEs may be linked to a response to ICIs and to longer survival in NSCLC patients (9-13), although studies 
focusing specifically on this relationship are yet to be carried out.

The use of patient reported outcomes (PROs), to obtain more reliable and complete information on patients' state of health and quality of life, is of particular interest. Not all studies included reported this kind of evaluation. Hence specific tools for use in patients being treated with ICIs, which are not inexpensive (14), are not only useful but necessary.

PD-1 and PD-L1 inhibitors show particular differences in biochemical efficacy. PD-L1 inhibitors do not block binding of the other PD-1 ligand, PD-L2, to the receptor, an interaction which generates inhibitory signals affecting the immune response. Furthermore, PD-L2 also binds to repulsive guidance molecule $b$ (RGMb), which regulates respiratory immunity (15). This may explain why the incidence of some AEs, such as pneumonitis, is different in treatment with PD-1 inhibitors than with PD-L1 inhibitors (16). In addition, the review published by Pillai et al. is not an individual participant data-based meta-analysis, as the authors have stated (7). This means that it is not possible to establish potential risk factors for a specific toxic effect, including for example the basal function of endocrine glands or the possible presence of concomitant pathologies such as pulmonary interstitial disease. The CheckMate 153 phase IIIb/IV study with nivolumab, involving a large sample of patients mostly belonging to a real-world population (also with Performance Status 2 and comorbidities), was included in the analysis with the incidence of grade 3-5 AEs as primary endpoint (17). However, this is only 1 phase IIIb/IV study included, and this could be a further risk of bias.

Immunotherapy certainly represents a revolutionary approach to treatment in the field of thoracic oncology, but there are still few data in the literature regarding possible differences between PD-1 and PD-L1 inhibitors in terms of toxicity. Since no direct comparison has been made between different ICIs, when activity and tolerability are similar it is impossible to recommend the use of one drug rather than another. As the number of patients treated with ICIs will continue to grow, efforts must be made to identify possible predictive biomarkers for response and toxicity beyond the PD-L1 expression, also with a view to the combination strategies which will conceivably be developed in the coming years. In current clinical practice there is undoubtedly a necessity for more widespread collaboration and a continuous collection of real-world data in order to facilitate therapeutic decision making when faced with patients who are often very different to those participating in clinical trials.

\section{Acknowledgements}

None.

\section{Footnote}

Conflicts of Interest: C Gridelli received honoraria as speaker bureau and advisory board member for BMS, MSD, Astra Zeneca. A Spagnuolo has no conflicts of interest to declare.

\section{References}

1. Topalian SL, Drake CG, Pardoll DM. Targeting the PD-1/B7-H1(PD-L1) pathway to activate anti-tumor immunity. Curr Opin Immunol 2012;24:207-12.

2. Barber DL, Wherry EJ, Masopust D, et al. Restoring function in exhausted CD8 T cells during chronic viral infection. Nature 2006;439:682-7.

3. Chen DS, Mellman I. Oncology meets immunology: the cancer-immunity cycle. Immunity 2013;39:1-10.

4. Khoja L, Day D, Wei-Wu Chen T, et al. Tumour- and class-specific patterns of immune-related adverse events of immune checkpoint inhibitors: a systematic review. Ann Oncol 2017;28:2377-85.

5. Michot JM, Bigenwald C, Champiat S, et al. Immunerelated adverse events with immune checkpoint blockade: a comprehensive review. Eur J Cancer 2016;54:139-48.

6. Champiat S, Lambotte O, Barreau E, et al. Management of immune checkpoint blockade dysimmune toxicities: a collaborative position paper. Ann Oncol 2016;27:559-74.

7. Pillai RN, Behera M, Owonikoko TK, et al. Comparison of the Toxicity Profile of PD-1 Versus PD-L1 Inhibitors in Non-Small Cell Lung Cancer: A Systematic Analysis of the Literature. Cancer 2018;124:271-7.

8. Puzanov I, Diab A, Abdallah K, et al. Managing toxicities associated with immune checkpoint inhibitors: consensus recommendations from the Society for Immunotherapy of Cancer (SITC) Toxicity Management Working Group. J Immunother Cancer 2017;5:95.

9. Naidoo J, Wang X, Woo KM, et al. Pneumonitis in patients treated with anti-programmed Death-1/ programmed death ligand 1 therapy. J Clin Oncol 2017;35:709-17.

10. Osorio JC, Ni A, Chaft JE, et al. Antibody-mediated thyroid dysfunction during T-cell checkpoint blockade in patients with non-small-cell lung cancer. Ann Oncol 2017;28:583-9.

11. Owen DH, Wei L, Villalona-Calero MA, et al. Impact of 
immune-related adverse events (irAE) on overall survival (OS) in patients treated with immunotherapy for non-small cell lung cancer (NSCLC). J Clin Oncol 2017;35:abstr 9080.

12. Haratani K, Hayashi H, Chiba Y, et al. Association of Immune-Related Adverse Events With Nivolumab Efficacy in Non-Small Cell Lung Cancer. JAMA Oncol 2018;4:374-8.

13. Teraoka S, Fujimoto D, Morimoto T, et al. Early ImmuneRelated Adverse Events and Association with Outcome in Advanced Non-Small Cell Lung Cancer Patients Treated with Nivolumab: A Prospective Cohort Study. J Thorac Oncol 2017;12:1798-805.

14. Tartari F, Santoni M, Burattini L, et al. Economic sustainability of anti-PD-1 agents nivolumab and pembrolizumab in cancer patients: recent insights and

Cite this article as: Spagnuolo A, Gridelli C. "Comparison of the toxicity profile of $\mathrm{PD}-1$ versus $\mathrm{PD}-\mathrm{L} 1$ inhibitors in nonsmall cell lung cancer": is there a substantial difference or not? J Thorac Dis 2018;10(Suppl 33):S4065-S4068. doi: 10.21037/ jtd.2018.09.83 future challenges. Cancer Treat Rev 2016;48:20-4.

15. Xiao Y, Yu S, Zhu B, et al. RGMb is a novel binding partner for PD-L2 and its engagement with PD-L2 promotes respiratory tolerance. J Exp Med 2014;211:943-59.

16. Khunger $M$, Rakshit $S$, Pasupuleti V, et al. Incidence of Pneumonitis With Use of Programmed Death 1 and Programmed Death-Ligand 1 Inhibitors in Non-Small Cell Lung Cancer: A Systematic Review and MetaAnalysis of Trials. Chest 2017;152:271-81.

17. Bauer TM, McCleod M, Chandler JC, et al. An ongoing phase IIIb/IV safety trial of nivolumab (NIVO) in patients (pts) with advanced or metastatic non-small-cell lung cancer (NSCLC) who progressed after receiving 1 or more prior systemic regimens. J Clin Oncol 2015;33:abstr 3013. 